\title{
Patient Perspectives on DMARD Safety Concerns in Rheumatology Trials: Results from Inflammatory Arthritis Patient Focus Groups and OMERACT Attendees Discussion
}

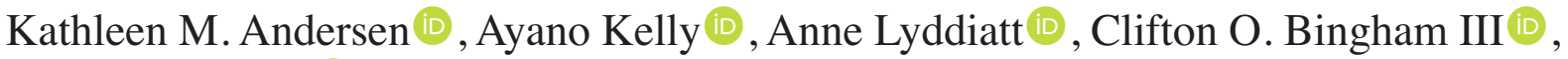 \\ Vivian P. Bykerk (D), Adena Batterman, Joan Westreich, Michelle K. Jones, Marita Cross, \\ Peter M. Brooks (1), Lyn March (1), Beverley Shea ${ }^{(1)}$, Peter Tugwell ${ }^{(}$, Lee S. Simon (1), \\ Robin Christensen (i), and Susan J. Bartlett (1)
}

\begin{abstract}
Objective. The Outcome Measures in Rheumatology (OMERACT) Safety Working Group is identifying core safety domains that matter most to patients with rheumatic disease.

Methods. International focus groups were held with 39 patients with inflammatory arthritis to identify disease-modifying antirheumatic drug (DMARD) experiences and concerns. Themes were identified by pragmatic thematic coding and discussed in small groups by meeting attendees.

Results. Patients view DMARD side effects as a continuum and consider the cumulative effect on day-to-day function. Disease and drug experiences, personal factors, and life circumstances influence tolerance of side effects and treatment persistence.

Conclusion. Patients weigh overall adverse effects and benefits over time in relation to experiences and life circumstances. (First Release April 15 2019; J Rheumatol 2019;46:1168-72; doi:10.3899/ jrheum.181185)
\end{abstract}

Key Indexing Terms: OMERACT CLINICAL TRIALS

DRUG TOXICITY RISK PATIENT SATISFACTION DISEASE-MODIFYING ANTIRHEUMATIC DRUGS
From the Department of Family Medicine, and the Department of Medicine, McGill University, Montreal, Quebec; Ottawa Hospital Research Institute, Ottawa Hospital; Division of Rheumatology, Department of Medicine, and School of Epidemiology and Public Health, Faculty of Medicine, University of Ottawa; Clinical Epidemiology Program, Ottawa Hospital Research Institute, Ottawa, Ontario, Canada; Musculoskeletal Statistics Unit, The Parker Institute, Bispebjerg and Frederiksberg Hospital, Copenhagen; Department of Rheumatology, Odense University Hospital, Odense, Denmark; Canberra Rheumatology; College of Health and Medicine, Australian National University, Canberra; Centre for Kidney Research, The Children's Hospital at Westmead; Institute of Bone and Joint Research-Kolling Institute, University of Sydney; Sydney Medical School, University of Sydney, Sydney; Department of Rheumatology, Royal North Shore Hospital; Institute of Bone and Joint Research, Kolling Institute, Northern Sydney Local Health District, St Leonards; Centre for Health Policy, School of Population and Global Health, The University of Melbourne, Melbourne; Northern Health, Epping, Australia; Division of Rheumatology,

Department of Medicine, Johns Hopkins University, Baltimore, Maryland; Division of Rheumatology, Department of Medicine, Hospital for Special Surgery; Department of Social Work Programs, Hospital for Special Surgery, New York, New York; SDG LLC, Cambridge, Massachusetts, USA.

OMERACT is an organization that develops and validates outcome measures in rheumatology randomized controlled trials and longitudinal observational studies and receives arms-length funding from 12 sponsors. $R C$ is supported by a core grant from the Oak Foundation (OCAY-13-309). $C O B / M K J$ and this work supported in part by US National Institutes of Health NIH-AR072574 Core B, and the Camille Julia Morgan Arthritis Research and Education Fund.
K.M. Andersen, MSc, Department of Family Medicine, McGill University, and Musculoskeletal Statistics Unit, The Parker Institute, Bispebjerg and Frederiksberg Hospital; A. Kelly, MD, PhD student, Canberra

Rheumatology, and College of Health and Medicine, Australian National University, and Centre for Kidney Research, The Children's Hospital at Westmead; A. Lyddiatt, Patient Partner; C.O. Bingham III, MD, Division of Rheumatology, Department of Medicine, Johns Hopkins University; V.P. Bykerk, MD, Division of Rheumatology, Department of Medicine, Hospital for Special Surgery; A. Batterman, MSW, Department of Social Work Programs, Hospital for Special Surgery; J. Westreich, MSW, Department of Social Work Programs, Hospital for Special Surgery; M.K. Jones, BSc, Division of Rheumatology, Department of Medicine, Johns Hopkins University; M. Cross, PhD, Institute of Bone and Joint Research-Kolling Institute, University of Sydney, and Rheumatology Department, Royal North Shore Hospital; P.M. Brooks, MD, Centre for Health Policy, School of Population and Global Health, The University of Melbourne, and Northern Health; L. March, MBBS, MSc, PhD, FRACP, FAFPHM, Sydney Medical School, University of Sydney, and Institute of Bone and Joint Research, Kolling Institute, Northern Sydney Local Health District, St Leonards, and Department of Rheumatology, Royal North Shore Hospital; B. Shea, PhD, Clinical Investigator, Ottawa Hospital Research Institute, Ottawa Hospital, and Adjunct Professor, School of Epidemiology and Public Health, Faculty of Medicine, University of Ottawa; P. Tugwell, MD, MSc, Division of Rheumatology, Department of Medicine, and School of Epidemiology and Public Health, Faculty of Medicine, University of Ottawa, and Clinical Epidemiology Program, Ottawa Hospital Research Institute; L.S. Simon, MD, SDG LLC; $R$. Christensen, PhD, Professor of Biostatistics and Clinical Epidemiology; Musculoskeletal Statistics Unit, The Parker Institute, Bispebjerg and Frederiksberg Hospital, and Department of Rheumatology, 
Many drugs used in rheumatology carry substantial benefit and potential harms. While medication-related symptoms and adverse events are prevalent in rheumatology randomized controlled trials (RCT), clinicians frequently underestimate their severity and focus on different priorities than patients when judging the effectiveness of medications ${ }^{1}$. The full spectrum and combination of potential harms are important to identify, given that adverse drug reactions cause considerable morbidity and mortality worldwide ${ }^{2}$.

Current approaches to safety monitoring depend heavily on healthcare professionals, despite known limitations including underreporting and discordant perspectives of patients and clinicians ${ }^{3}$. Although regulatory authorities in Europe and the United States call for inclusion of patient-reported information of benefit and safety, there is limited understanding of what matters most to patients regarding disease-modifying antirheumatic drug (DMARD) safety. While a patient-based reporting system has been developed to identify symptoms and adverse events from patients in cancer clinical trials ${ }^{4}$, to our knowledge, no standardized approach currently exists for rheumatology trials. To date, there has been little effort to systematically collect information directly from patients about side effects that concern them.

Patients frequently report they experience side effects including upset stomach, fatigue, nausea, and gastrointestinal distress when taking medicines for rheumatic diseases ${ }^{5,6,7,8,9}$. Symptomatic adverse events, also known as side effects, are increasingly recognized as an important contributor to poor adherence and can lead to patient-initiated dose reduction and early discontinuation. Rheumatology RCT require substantial time, effort, and resources, and rely on patient altruism. It is essential to assess the range of potential benefits and harms associated with an intervention and to include safety outcomes that are relevant and meaningful to patients. The Outcome Measures in Rheumatology (OMERACT) Safety Group was reestablished at OMERACT 2016 to identify core domains for safety aspects that matter most to patients in rheumatology trials ${ }^{10}$.

\section{MATERIALS AND METHODS}

As part of a larger mixed-methods study, we present here the qualitative findings from our a priori protocol, which was registered in March 2017 with the Core Outcome Measures in Effectiveness Trials (http:// www.comet-initiative.org/studies/details/1120?result=true). An initial scoping review by this group in 2016 revealed that safety has differing connotations and reflects a spectrum of events. An optimal method and language to assess patient-valued safety aspects in trials has not been identified.

Odense University Hospital; S.J. Bartlett, PhD, Professor, Department of

Medicine, McGill University, and Adjunct Professor, Division of

Rheumatology, Department of Medicine, Johns Hopkins University.

Address correspondence to S.J. Bartlett, McGill University, 5252

Boulevard de Maisonneuve Ovest, \#3D.57, Montreal, Quebec H4A 3S5,

Canada.E-mail: susan.bartlett@mcgill.ca

Accepted for publication January 31, 2019.
To gain insight into patient priorities, 6 semistructured focus groups were held with patients with inflammatory arthritis (IA) in Canada, the United States, and Australia from March-May 2018, facilitated by an experienced qualitative researcher. According to a prespecified interview schedule, participants were encouraged to describe their experiences taking DMARD for their arthritis, as well as perceptions of benefit and potential harm. Ethical approval for the focus groups was obtained (Bingham: NA00066663, Bykerk: 2018-0233, Bartlett: 2018-4404, Kelly: LNR/16/LPOOL/701 and LNRSSA/17/HAWKE/429). Groups were recorded and transcribed. We conducted a targeted and pragmatic analysis based in grounded theory to descriptively and thematically summarize discussions ${ }^{11}$.

Initial results were presented at the OMERACT Pre-Meeting (Improving Risk-Benefit Assessment of Drugs with an Emphasis on Patients and Their Perspective) on May 13 and 14, 2018, in Terrigal, New South Wales, Australia (described elsewhere ${ }^{12}$ ). Attendees broke into 5 groups; seating was prearranged to ensure the inclusion of diverse perspectives and individuals within groups. Patient research partners (PRP) reported results of these discussions to all attendees at a report-back session.

\section{RESULTS}

Thirty-nine adults with IA participated in the initial focus groups from the United States (12 women, 2 men: Maryland and New York), Canada (8 women, 2 men: Ontario, Quebec, and Manitoba), and Australia (11 women, 4 men: New South Wales).

In brief, 4 themes were identified (Table 1). First, almost all participants reported experiencing many DMARD side effects that are often termed "nuisance side effects." Although nuisance side effects are often not viewed by trialists and treating clinicians as problematic, patients reported they had a considerable cumulative effect on quality of life but were seen as "the price you pay" for improvement. Almost all patients indicated that the effect on day-to-day physical and social function mattered more to them than physiologic manifestations. Most learned to live with DMARD side effects; however, some lives were completely changed. Many reported that the disabling and persistent side effects were managed using a range of behavioral and nutritional strategies (e.g., talk less to avoid irritating a mouth ulcer, exercise, earlier bedtime, reduced participation, yogurt, antidiarrheal over-the-counter medications) to attenuate common side effects (e.g., gastrointestinal distress, mouth ulcers, fatigue). The failure of self-management and the considerable cumulative burden of side effects led to increased frustration and helplessness; these were key reasons some decided to discontinue a medication.

Second, almost all patients reported difficulty with symptom attribution, and would only identify medications as a potential cause after first considering lifestyle, current health, and other life circumstances. Within each group, patients were often surprised when others described side effects ("I didn't know that could be a side effect. I noticed my drain was full of hair, but never thought it might be the medication.") Many patients were uncomfortable discussing side effects to trialists or providers out of concern they would be labeled as "whiners," removed from a trial, or be switched to an inferior drug.

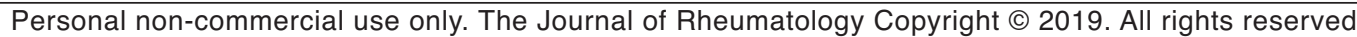


Table 1. Themes and illustrative quotes identified in inflammatory arthritis participants regarding DMARD safety concerns.

Theme Illustrative Quote

Patients and clinicians view side effects differently;

"nuisance" side effects persist and can have a substantial cumulative effect and often lead to patient-initiated dose reductions and discontinuation

Patients have difficulty sorting out side effects from other factors

Different DMARD elicit different safety concerns

Concerns are influenced by disease and medication experiences, and individual and social factors
"I feel like I can't think anymore, and that really affects work and that's my biggest problem. I can push through the pain and I can sometimes push through the fatigue, but I can't think clearly. I just can't do my job, and that's been the biggest struggle for me." - Female, USA

"When you do bring up a concern like 'Well, I've got really bad headaches'... And [MD says] 'Oh, well, that's a hard problem to deal with.' It gets kind of sloughed off." - Female, Canada

"Started out with nausea, and for a long time it wasn't too bad. But the last 3 years, I just felt nauseated 24/7, even with the injection - nausea, headache, digestive issues. And I just took myself off it... and I feel a whole heck of a lot better." - Female, Canada

"I thought it can't be the medication. I didn't eat properly today. I've had 13 cups of coffee. I need to go home and get some food in me... and all kinds of reasons for what was happening, other than [the medication]. [Long pause] It was the medication." - Female, Canada

"Honestly, sometimes I don't think about it... I would not have said to you, 'Oh, I have lost my hair.' But you know what? I'm cleaning out my drain... I'm cleaning a lot of hair out of my drain. But I wouldn't think to report it." - Female, USA

"[I worry about] bad things that can happen in you that you can't see." - Male, Canada

"....and then [with] going through all the side effects, but you want me to take this forever? You know, what, I'm 26 and I'm supposed to just take this forever now even though you've told me the effects it's going to have [on] my liver, etc.? ...I am not satisfied with just taking the medications that they're giving me for however long. I need to know there's some sort of end date." - Female, Australia

"When I first was diagnosed, I didn't think I was going to live as long as I did. That's how bad I felt. So, the side effects...just have to step aside right now. Because I look at the positive part of it. I can walk 4 blocks and it don't bother me." - Female, USA

"I'm definitely worried about the longterm effects... If I wanted to have a family, how would I do that? ...And the doctors always saying that that's going to be fine and manageable. And I know that obviously other people do it. ...But it is something that I think about." - Female, Australia

DMARD: disease-modifying antirheumatic drug.

Third, participants reported that different drugs elicited different safety concerns. Methotrexate (MTX), often the first treatment, was perceived as the most worrisome; several noted that the initiation of MTX as a first-line treatment often resulted in toxicity concerns being emphasized when the patient is still coping with accepting their diagnosis. Patients noted that while their physicians embrace MTX use, patients are often initially terrified to use it. In all groups, MTX was largely viewed as a common enemy, uniting the group. Conversely, the first mention of steroid use divided groups into 2 camps, eliciting strong opinions that were either testimonies to the benefits or admonitions against use. One patient noted "prednisone is the new smoking," reflecting both a perceived stigma and difficulty discontinuing once started. Drug switches, particularly when treatment was escalated to include biologics, were embraced by patients when their disease was poorly controlled. However, switches to generic medications when disease was well controlled were stressful and evoked concerns about loss of control. Safety concerns for DMARD are influenced by disease and medication experiences, personal and life circumstances, and exposure to stories from other patients.

Fourth, participants described how they weighed the safety versus effectiveness of DMARD to decide whether the medication was optimal for them (Figure 1). Patients with higher safety concerns tended to be younger, had more recent onset of IA, had tried fewer medications, returned more quickly to prediagnosis function with treatment, and had not experienced a major relapse. Conversely, patients who were willing to tolerate lower perceived levels of drug safety in favor of higher effectiveness tended to have longstanding disease, greater disability, and greater improvement in function with treatment. Patient characteristics (current living situation and roles), disease experience (e.g., severity of disease at its worst, number of failed medications), and social modeling (e.g., reports on social media) influenced the way in which patients viewed adverse events and side effects.

\section{DISCUSSION}

These results generated considerable discussion among premeeting attendees. Many of the PRP had strong emotional reactions during the presentation, and almost all reported feeling validated and reassured that these issues were being acknowledged. Several attendees reported that these results highlighted the cumulative effect and interference of treatments as more meaningful to patients than the discrete adverse events that are typically identified in drug trials (i.e., physiological manifestations), suggesting a paradigm shift may be warranted.

Several themes emerged in the report-backs. There was 


\section{Higher importance on effectiveness}

\section{Higher safety concerns}

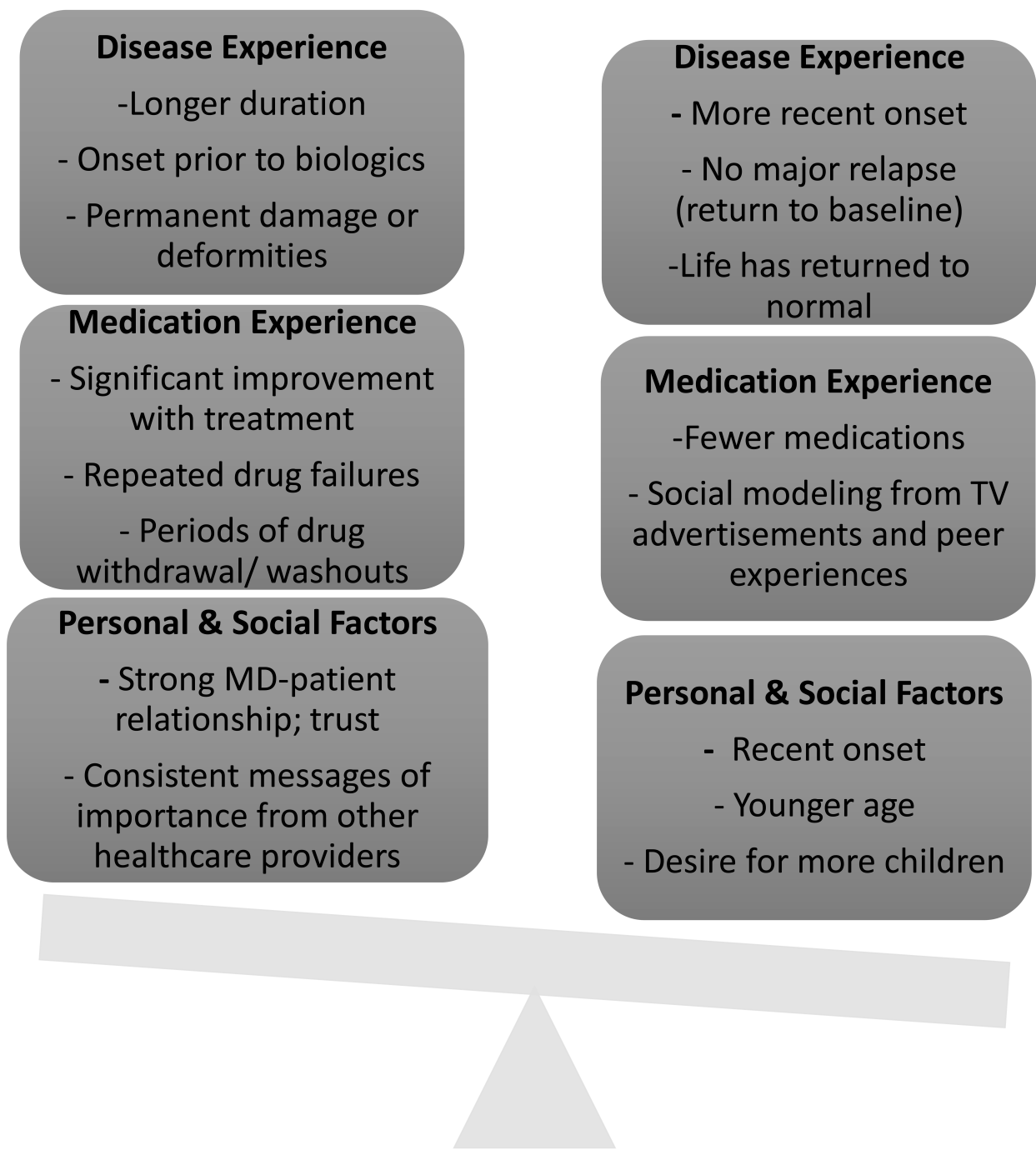

Figure 1. The dynamic balance of safety and effectiveness concerns among people with inflammatory arthritis, which can shift over time in response to many factors.

consensus that the current dichotomization of side effects (i.e., "nuisance" vs important) is judgmental and often arbitrary, potentially stigmatizing to patients, and does not reflect patient priorities. Some note that clinicians often use the term "nuisance side effects" to help diminish patient concerns; there is also discomfort discussing side effects when it is unclear how they can be mitigated. There was consensus that further research is needed to identify patient-relevant questions on drug safety concerns and ways to create conversations that encourage and support broader discussion. In clinical trials, it will be important to measure not only how an individual feels and functions, but also the effect on everyday life (e.g., "How is the medication affecting you? What have you or others noticed since starting the drug?"). Given that current life circumstances play a major role in the patient's ability and willingness to tolerate specific

Personal non-commercial use only. The Journal of Rheumatology Copyright (C) 2019. All rights reserved. 
side effects (e.g., fatigue, nausea, diarrhea), there is a need to measure and incorporate contextual factors when interpreting results of drug trials. Future trials and longitudinal studies should query patient satisfaction with the medication and move toward a systematic and standardized approach to identify this important data. For example, in oncology, the 5 "WIWI Questions" ("Was it worth it? Would you do again? Did quality of life improve? How satisfied are you with outcome? Would you recommend to others?") have been used to assess how patients view the benefits and costs of treatment.

Our findings and discussion among attendees prompted discussions within OMERACT and modification of the OMERACT Filter ${ }^{13,14}$. Version 2.1 now explicitly includes "Benefits and Harms" as a potential core domain to identify both the intended and unintended effects of interventions. It is anticipated that this domain will be recommended for inclusion in many future OMERACT core domain sets. Additional work by the safety group in other countries is needed to confirm and extend these findings, and the results of this qualitative work will feed into future quantitative and qualitative phases (i.e., Delphi and consensus work) toward agreement on core domains.

\section{REFERENCES}

1. Basch $E$. The missing voice of patients in drug-safety reporting. N Engl J Med 2010;362:865-9.

2. World Health Organization. Safety of medicines: a guide to detecting and reporting adverse drug reactions: why health professionals need to take action. Geneva, Switzerland; 2002. [Internet. Accessed April 2, 2019.] Available from: https://apps.who.int/iris/handle/10665/67378

3. Banerjee AK, Okun S, Edwards IR, Wicks P, Smith MY, Mayall SJ, et al. Patient-reported outcome measures in safety event reporting: PROSPER consortium guidance. Drug Saf 2013;36:1129-49.

4. Dueck AC, Mendoza TR, Mitchell SA, Reeve BB, Castro KM, Rogak LJ, et al; National Cancer Institute PRO-CTCAE Study
Group. Validity and reliability of the US National Cancer Institute's patient-reported outcomes version of the common terminology criteria for adverse events (PRO-CTCAE). JAMA Oncol 2015;1:1051-9

5. D'Angelo S, Carriero A, Gilio M, Ursini F, Leccese P, Palazzi C. Safety of treatment options for spondyloarthritis: a narrative review. Expert Opin Drug Saf 2018;17:475-86.

6. Gawert L, Hierse F, Zink A, Strangfeld A. How well do patient reports reflect adverse drug reactions reported by rheumatologists? Agreement of physician- and patient-reported adverse events in patients with rheumatoid arthritis observed in the German biologics register. Rheumatology 2011;50:152-60.

7. Pasma A, van 't Spijker A, Luime JJ, Walter MJ, Busschbach JJ, Hazes JM. Facilitators and barriers to adherence in the initiation phase of disease-modifying antirheumatic drug (DMARD) use in patients with arthritis who recently started their first DMARD treatment. J Rheumatol 2015;42:379-85.

8. Linden C, Bjorklund A. Living with rheumatoid arthritis and experiencing everyday life with TNF- $\alpha$ blockers. Scand J Occup Ther 2010;17:326-34.

9. Goodacre LJ, Goodacre JA. Factors influencing the beliefs of patients with rheumatoid arthritis regarding disease-modifying medication. Rheumatology 2004;43:583-6.

10. Klokker L, Tugwell P, Furst DE, Devoe D, Williamson P, Terwee $\mathrm{CB}$, et al. Developing an OMERACT core outcome set for assessing safety components in rheumatology trials: the OMERACT safety working group. J Rheumatol 2017;44:1916-9.

11. Braun V, Clarke V. Using thematic analysis in psychology. Qual Res Psychol 2008;3:77-101.

12. Andersen KM, Cheah JT, March L, Bartlett SJ, Beaton D, Bingham CO III, et al. Improving benefit-harm assessment of therapies from the patient perspective: OMERACT premeeting toward consensus on core sets for randomized controlled trials. J Rheumatol 2019;46:1053-8.

13. Boers M, Kirwan JR, Tugwell P, Beaton D, Bingham CO III, Conaghan PG, et al. The OMERACT Handbook. [Internet. Accessed September 20, 2018.] Available from: https://omeract.org/resources

14. Boers M, Kirwan JR, Wells G, Beaton D, Gossec L, d'Agostino MA, et al. Developing core outcome measurement sets for clinical trials: OMERACT filter 2.0. J Clin Epidemiol 2014;67:745-53. 